\title{
Improving precision and accuracy of DTI experiments with the simplified BSD calibration - computer simulations
}

\author{
Karol Borkowski \\ Faculty of Physics and Applied Computer Science, \\ AGH University of Science and Technology \\ 30-059 Cracow, Poland \\ e-mail: Karol.Borkowski@fis.agh.edu.pl
}

\author{
Artur Krzyżak \\ Faculty of Geology, Geophysics and Environmental \\ Protection, \\ AGH University of Science and Technology \\ 30-059 Cracow, Poland
}

\begin{abstract}
The $b$-matrix spatial distribution (BSD) is an effective method of improving accuracy of diffusion tensor imaging (DTI) measurements. It is based on a calibration with an anisotropic phantom measured in six positions inside an MRI scanner. However, if the contribution of non-diffusion gradients to the $b$-matrix can be neglected, simplified form of calibration with only 3 positions of the phantom could be sufficient. We called this approach simplified BSD-DTI (sBSDDTI).

In this paper we introduce the above-mentioned technique and present the results of the computer simulations of BSD-DTI experiments and compare them with standard DTI. The complete BSD method, sBSD as well as BSD with assumption of phantom uniformity (uBSD) were simulated.

The simulations revealed that both simplified methods are less accurate than the complete BSD-DTI. Nevertheless, the calibration procedures and the algorithms are streamlined.
\end{abstract}

\section{INTRODUCTION}

$\mathrm{D}$ iffusion tensor imaging (DTI) is a powerful MRI technique with multiple clinical applications, including presurgical planning and intraoperative guidance in regions adjacent to critical neural tracts, evaluation and treatment of neurodegenerative diseases like epilepsy, multiple sclerosis, Alzheimer's or ischemic stroke [1]. Its accuracy depends strictly on correct identification of the $b$-matrix for a given imaging sequence [2][3]. Due to the complex character of the $b$-matrix and the fact that it is not constant across the volume [2], its analytical derivation is a very tedious and imprecise process [4][5]. Therefore, in the majority of MRI systems, the $b$-matrix is calculated relying only on the diffusion gradient vectors.

Several ways of improving the precision of derivation of the correct form of the $b$-matrix have been reported. Some of them take into account the cross-terms between the diffusion and imaging gradients. That includes refocusing each imaging gradient before turning on the diffusion gradient and refocusing each diffusion gradient before the imaging gradient is applied [6] or acquiring data twice; once with the

Research financed by the National Centre of Research and Development, contract. No. PBS2/A2/16/2013 and contract No. STRATEGMED2/265761/10/NCBR/2015. given diffusion gradient and once with the opposite polarity [7][8]. However, these methods involve a prolonged acquisition time. The cross-terms effect can be also minimized by numerical calculations of the $b$-matrix [9][3], using calibration scans of an isotropic phantom [10] or by establishing the optimal diffusion gradient scheme [11].

In all of the above-mentioned techniques only diffusion, imaging and other known gradients are taken into account, while the background noises and further unknown factors, which may have an impact on the $b$-matrix, are neglected. Moreover, all of these practices assume the same form of the b-matrix throughout the imaging space, what is not true in general.

The $b$-matrix Spatial Distribution in Diffusion Tensor Imaging (BSD-DTI) is a method which enables one to discover the real form of the $b$-matrix for every voxel using a precisely defined anisotropic phantom [2]. It allows to maintain the accuracy and precision of the measurement even if the diffusion properties of the phantom are not spatially constant [12].

In this paper the simplified form of the BSD calibration is introduced. It requires measurement of the phantom in only three positions instead of six. Moreover, we present the results of the computer simulations which depict the effectiveness of this method.

\section{THEORY}

\section{A. Diffusion Tensor Calculation}

The elements of a diffusion tensor can be calculated from the formula: [13]

$\ln \left(\frac{S_{n}(b)}{S\left(b_{0}\right)}\right)=-\sum_{i, j=1}^{3}\left(b_{i j}-b_{0 i j}\right) D_{i j}$

which is the form of the Stejskal-Tanner equation, where $S_{n}(b)$ is a signal intensity for the nth diffusion gradient, $S\left(b_{0}\right)$ is a signal intensity without any diffusion gradient.

$D_{i j}$ and $b_{i j}$ are the components of the diffusion tensor and the $b$-matrix, respectively.

\section{B. B matrix calculation}

The $b$-matrix used in eq. (1) is given by [9] 


$$
b=\int_{0}^{2 \tau}[k(t)-2 H(t-\tau) k(\tau)][k(t)-2 H(t-\tau) k(\tau)]^{T} d t,(2 \mathrm{a})
$$

where

$$
k(t)=\gamma \int_{0}^{t} G\left(t^{\prime}\right) d t^{\prime}
$$

$G(t)$ is a column vector which represents the particular gradient in the imaging sequence. $\gamma$ is the gyromagnetic ratio, $2 \tau$ is the echo time, $H(t)$ is the Heaviside unit-step function.

If the imaging and other background gradients can be ignored, the $b$-matrix can be calculated from the dyadic product of the adequate gradients [14]; for example

$$
\begin{aligned}
b_{n}=G_{n} G_{n}{ }^{T} & =\left[\begin{array}{l}
g_{x} \\
g_{y} \\
g_{z}
\end{array}\right]\left[\begin{array}{lll}
g_{x} & g_{y} & g_{z}
\end{array}\right]= \\
& =\left[\begin{array}{ccc}
g_{x}{ }^{2} & g_{x} g_{y} & g_{x} g_{z} \\
g_{x} g_{y} & g_{y}{ }^{2} & g_{y} g_{z} \\
g_{x} g_{z} & g_{y} g_{z} & g_{z}{ }^{2}
\end{array}\right]
\end{aligned}
$$

Otherwise, the expressions for components of the $b$-matrix, due to the cross-terms between diffusion and other gradients, has more complex form:

$b_{i i}=a G_{i}^{2}+b G_{i}+c$

for the diagonal components and

$$
b_{i j}=a G_{i} G_{j}+b G_{i}+c G_{j}+d
$$

for the off-diagonal.

\section{Calibration with anisotropic phantom in three positions}

To perform the simplified BSD-DTI (sBSD) calibration the phantom is situated inside an MRI scanner in such a way, that its diffusion tensor is diagonal in the laboratory coordinate system and then the diffusion tensor is measured. Subsequently, the phantom is rotated twice about $90^{\circ}$ around the two orthogonal axes of the coordinate system respectively and each time the measurement is repeated. In this situation, according to the Stejskal-Tanner equation, the signal attenuation in voxel $(k, l, m)$ in laboratory coordinate frame for a particular diffusion gradient is given by

$$
\begin{aligned}
\ln \left(\frac{S\left(b_{k l m}\right)}{S(0)}\right) & =-b_{k l m}:\left[\begin{array}{ccc}
D 1_{k l m} & 0 & 0 \\
0 & D 2_{k l m} & 0 \\
0 & 0 & D 3_{k l m}
\end{array}\right]= \\
& =b_{x x, k l m} D 1_{k l m}+b_{y y, k l m} D 2_{k l m}+b_{z z, k l m} D 3_{k l m}
\end{aligned}
$$

Providing that diffusion properties of the phantom are well known in a coordinate system associated with the phantom $(r, s, t)$, in laboratory coordinate frame $(k, l, m)$ they can be found in two steps [15]. First, the $(k, l, m)$ coordinates are found by

$$
\left[\begin{array}{l}
k \\
l \\
m
\end{array}\right]=R(\alpha, \beta, \gamma) \cdot\left[\begin{array}{l}
r \\
s \\
t
\end{array}\right],
$$

where $R(\alpha, \beta, \gamma)$ is a rotation matrix.

In the next step the diffusion tensor $D_{k l m}$ is derived by rotation transformation

$$
=R(\alpha, \beta, \gamma) \cdot D_{r s t} \cdot R^{T}(\alpha, \beta, \gamma) \text {. }
$$

In order to calculate the diagonal components of the $b$-matrix, one must solve the system of three equations for every diffusion gradient direction. Assuming that $b$-matrix has a form given by Eq. (3), off-diagonal components are equal to:

$b_{i j}=\operatorname{sgn}\left(g_{i} g_{j}\right) \sqrt{b_{i i} b_{j j}}$.

where $\operatorname{sgn}\left(g_{i} g_{j}\right)$ is a sign of the product $g_{i} g_{j}$, where $g_{\mathrm{i}}$ and $g_{\mathrm{j}}$ are the diffusion gradient strengths in $i$ and $j$ direction, respectively.

If the diffusion properties of a phantom are assumed to be constant in its whole volume, the $k, l$ and $m$ indexes of the tensor $D$ can be removed. BSD calibration under such assumption has been called uniform BSD-DTI (uBSD-DTI) [7].

\section{Materials AND Methods}

In order to examine the effectiveness of simplified BSDDTI method in comparison to BSD-DTI, uniform BSD-DTI (uBSD-DTI) and standard DTI (S-DTI), the computer simulations were conducted. First of all, the expressions given by Eq. (4a) and (4b) were derived for an exemplary imaging protocol for Bruker BioSpin 9.7 $\mathrm{T}$ imaging system. Components of $b$-matrices are given by

$b_{x x}=598.5 G_{x}^{2}+73.5 G_{x}+12.5$,

$b_{y y}=600 G_{y}^{2}+72 G_{y}+12.5$,

$b_{z z}=596 G_{z}^{2}+76 G_{z}+13$,

$b_{x y}=597 G_{x} G_{y}+37 G_{x}+37 G_{y}+12.5$,

$b_{x z}=597.5 G_{x} G_{z}+38 G_{x}+37 G_{z}+13$,

$b_{y z}=598.5 G_{y} G_{z}+37.5 G_{y}+36 G_{z}+13$.

Diffusion gradient directions were chosen as follows

$$
\begin{aligned}
& G_{1}=[0.666,0.333,0.666], \\
& G_{2}=[0.666,-0.333,0.666], \\
& G_{3}=[0.333,0.666,0.666],
\end{aligned}
$$


$G_{4}=[-0.333,0.666,0.666]$,

$G_{5}=[0.666,0.666,0.333]$,

$G_{6}=[0.666,0.666,-0.333]$.

Then, the $b$-matrix spatial distribution was established by distorting the diffusion gradients and calculating $b$ matrices for every voxel separately using Eqs. (9a)-(9f). The gradients were distorted systematically in following way

$G_{n, x y z=}^{\prime} G_{n} \sigma(x+y+z-36) / 12.4904$,

where $G_{n, x y z}^{\prime}$ is a distorted diffusion gradients for a voxel $(x, y, z)$ in the laboratory coordinate system. $G_{n}$ is a diffusion gradient in nth direction. $\sigma$ is the relative standard deviation of the spatial gradient distribution. In described simulations this parameter was set equal to $5 \%$.

Subsequently, simulations of the BSD-DTI calibrations were performed for a virtual anisotropic phantom. The phantom was a cube with a side equal to 41 voxels. This size is required to indicate the $b$-matrix spatial distribution in the $25 \times 25 \times 25$ voxels field of view (FOV). Diffusion properties of the phantom were similar to the diffusion properties of the anisotropic plate phantom [16]. The mean eigenvalues of its diffusion tensor were $0.002,0.002$ and $0.0005 \mathrm{~mm}^{2} / \mathrm{s}$. In order to imitate the properties of a real phantoms the tensor was spatially distorted by Gaussian noise with relative standard deviation equal to $1 \%$ and mean value equal to 0 . The distribution of the $b$-matrix was calculated in four ways. Using the complete BSD-DTI method, uniform BSD, simplified BSD and simplified uniform BSD (uniform BSD with three phantom's positions - usBSD). Finally, diffusion tensor experiment were simulated for other two virtual homogeneous phantoms $\mathrm{P} 1$ and $\mathrm{P} 2$. The former was characterized by diffusion tensor with eigenvalues equal to $0.001,0.002$ and $0.003 \mathrm{~mm}^{2} / \mathrm{s}$. The latter was isotropic with diffusion coefficient equal to $0.002 \mathrm{~mm}^{2} / \mathrm{s}$. The first phantom was orientated in such a way that in the laboratory coordinate system its edges were inclined at $45^{\circ}$ angle from the axes of the laboratory coordinate system. The tensor was calculated using each of the aforementioned $b$-matrix spatial distributions, spatially constant $b$-matrix (S-DTI) and distribution calculated with approximation given by Eq. (3).

\section{RESULTS}

Tables 1 and 2 report the $b$-matrix elements calculated according to expressions (3) and (9a)-(9f), while table 3 reports the relative difference between them.

Mean values and relative standard deviations of diffusion tensor eigenvalues obtained in the simulations are reported in table 4.

In the case of isotropic phantom P2 as well as the phantom P1 orientated in such a way that its diffusion tensor was diagonal and in the case of $b$-matrix calculated with dyadic approximation, obtained results were similar for the simplified BSD and the BSD-DTI approaches.

Computing time of the $b$-matrix spatial distribution calculations performed on a personal computer was 331s, 227s, 72s and 44s for BSD-DTI, uBSD, sBSD and usBSD, respectively.

TABLE I.

B-MATRIX ELEMENTS CALCULATED ACCORDINGLY TO EQS. 9A - 9F.

\begin{tabular}{|l|l|l|l|l|l|l|}
\hline & bxx & byy & bzz & bxy & bxz & byz \\
\hline G1 & 315.00 & 90.67 & 315.56 & 169.67 & 315.56 & 169.50 \\
\hline G2 & 315.00 & 42.67 & 315.56 & -120.33 & 315.56 & -121.50 \\
\hline G3 & 91.00 & 314.67 & 315.56 & 169.67 & 170.11 & 315.00 \\
\hline G4 & 42.00 & 314.67 & 315.56 & -120.33 & -120.78 & 315.00 \\
\hline G5 & 315.00 & 314.67 & 91.56 & 314.67 & 170.44 & 170.00 \\
\hline G6 & 315.00 & 314.67 & 40.89 & 314.67 & -119.78 & -120.00 \\
\hline
\end{tabular}

TABLE 1.

B-MATRIX ELEMENTS CALCULATED ACCORDING TO EQ. 3.

\begin{tabular}{|l|l|l|l|l|l|l|}
\hline & bxx & byy & bzz & bxy & bxz & byz \\
\hline G1 & 315.00 & 90.67 & 315.56 & 169,00 & 315,28 & 169,15 \\
\hline G2 & 315.00 & 42.67 & 315.56 & $-115,93$ & 315,28 & $-116,03$ \\
\hline G3 & 91.00 & 314.67 & 315.56 & 169,22 & 169,46 & 315,11 \\
\hline G4 & 42.00 & 314.67 & 315.56 & $-114,96$ & $-115,12$ & 315,11 \\
\hline G5 & 315.00 & 314.67 & 91.56 & 314,83 & 169,82 & 169,73 \\
\hline G6 & 315.00 & 314.67 & 40.89 & 314,83 & $-113,49$ & $-113,43$ \\
\hline
\end{tabular}

TABLE 2.

RELATIVE DIFFERENCE BETWEEN BOTH SETS OF B-MATRIX ELEMENTS.

\begin{tabular}{|l|l|l|l|l|l|l|}
\hline & bxx & byy & bzz & bxy & bxz & byz \\
\hline G1 & $0 \%$ & $0 \%$ & $0 \%$ & $0.39 \%$ & $0.09 \%$ & $0.21 \%$ \\
\hline G2 & $0 \%$ & $0 \%$ & $0 \%$ & $3.66 \%$ & $0.09 \%$ & $4.50 \%$ \\
\hline G3 & $0 \%$ & $0 \%$ & $0 \%$ & $0.26 \%$ & $0.38 \%$ & $-0.04 \%$ \\
\hline G4 & $0 \%$ & $0 \%$ & $0 \%$ & $4.46 \%$ & $4.68 \%$ & $-0.04 \%$ \\
\hline G5 & $0 \%$ & $0 \%$ & $0 \%$ & $-0.05 \%$ & $0.36 \%$ & $0.16 \%$ \\
\hline G6 & $0 \%$ & $0 \%$ & $0 \%$ & $-0.05 \%$ & $5.25 \%$ & $5.47 \%$ \\
\hline
\end{tabular}

TABLE 3.

AVERAGE EIGENVALUES $\left[\mathrm{MM}^{2} / \mathrm{s}\right]$, ITS RELATIVE DIFFERENCE $\Delta$ BETWEEN REAL AND MEASURED ONES AND RELATIVE STANDARD DEVIATION (RSD) ACROSS THE FOV FOR PHANTOM P1 OBTAINED BY SIMULATING THE S-DTI, BSD-DTI, UBSD, SBSD AND USBSD EXPERIMENTS.

\begin{tabular}{|l|l|l|l|l|l|}
\hline & S-DTI & $\begin{array}{l}\text { BSD- } \\
\text { DTI }\end{array}$ & uBSD & sBSD & usBSD \\
\hline av. E1 & $\begin{array}{l}1.0021 \mathrm{E}- \\
03\end{array}$ & 0.001 & $\begin{array}{l}9.9859 \mathrm{E}- \\
04\end{array}$ & $\begin{array}{l}9.9630 \mathrm{E}- \\
04\end{array}$ & $\begin{array}{l}9.9521 \mathrm{E}- \\
04\end{array}$ \\
\hline av. E2 & $\begin{array}{l}2.0048 \mathrm{E}- \\
03\end{array}$ & 0.002 & $\begin{array}{l}2.0002 \mathrm{E}- \\
03\end{array}$ & $\begin{array}{l}2.0137 \mathrm{E}- \\
03\end{array}$ & $\begin{array}{l}2.0118 \mathrm{E}- \\
03\end{array}$ \\
\hline av. E3 & $\begin{array}{l}3.0074 \mathrm{E}- \\
03\end{array}$ & 0.003 & $\begin{array}{l}3.0006 \mathrm{E}- \\
03\end{array}$ & $\begin{array}{l}3.0520 \mathrm{E}- \\
03\end{array}$ & $\begin{array}{l}3.0218 \mathrm{E}- \\
03\end{array}$ \\
\hline$\Delta \mathbf{E} 1$ & $0.206 \%$ & $0 \%$ & $-0.141 \%$ & $-0.372 \%$ & $-0.481 \%$ \\
\hline$\Delta \mathbf{E 2}$ & $0.240 \%$ & $0 \%$ & $0.011 \%$ & $0.682 \%$ & $0.586 \%$ \\
\hline
\end{tabular}




\begin{tabular}{|l|l|l|l|l|l|}
\hline$\Delta$ E3 & $0.245 \%$ & $0 \%$ & $0.021 \%$ & $1.705 \%$ & $0.721 \%$ \\
\hline RSD E1 & $9.10 \%$ & $0 \%$ & $3.16 \%$ & $0.03 \%$ & $0.49 \%$ \\
\hline RSD E2 & $9.81 \%$ & $0 \%$ & $1.62 \%$ & $0.08 \%$ & $4.19 \%$ \\
\hline RSD E3 & $9.89 \%$ & $0 \%$ & $1.18 \%$ & $0.19 \%$ & $3.67 \%$ \\
\hline
\end{tabular}

\section{DISCUSSION}

The calculations of the $b$-matrix for the exemplary imaging sequence show that neglecting the cross-terms leads to a systematic error in the off-diagonal elements up to $5.47 \%$.

As expected the BSD-DTI method ensures the highest accuracy and precision of DTI experiments, but also takes the longest period of computing time. However, in the case of post hoc image analysis it may be irrelevant.

Accuracy of the uBSD-DTI is almost as good as in the case of BSD-DTI. Nevertheless, due to not taking into account the imperfections of the phantom the precision is lower.

In the simplified BSD-DTI, due to the differences in $b$ matrix elements reported in tab. 3, precision is slightly lower. Nevertheless, in comparison with S-DTI the accuracy is significantly improved. It also allows to reduce the computing time.

When one combines the uniform and simplified BSD approaches, in comparison to complete BSD-DTI method, the accuracy and the precision definitely decrease but also the calculations time is drastically reduced.

If the diffusion tensor of imaged object is diagonal in laboratory coordinate system (like in the cases of isotropic media) the off-diagonal components of the $b$-matrix can be neglected, therefore the quality of sBSD-DTI is the same that of BSD-DTI.

\section{CONCLUSION}

BSD-DTI is a robust and effective method of improving precision and accuracy of DTI experiment. Nevertheless, sBSD approach can be an expedient trade-off between quality and simplicity, especially when non-diffusion gradient presents during the imaging sequence are negligible.

\section{ACKNOWLEDGMENT}

The work was financed by the National Centre of Research and Development, contract. No. PBS2/A2/16/2013 and contract No. STRATEGMED2/265761/10/NCBR/2015.

K.B. thanks Marian Smoluchowski Cracow Scientific Consortium - KNOW for the support

\section{REFERENCES}

[1] A. Lerner, M. Mogensen, P. Kim, M. Shiroishi, D. Hwang and M. Law, "Clinical Applications of Diffusion Tensor Imaging", World Neurosurgery, vol. 82, no. 1-2, pp. 96-109, 2014, doi: 10.4103/09713026.38505

[2] A. Krzyżak and Z. Olejniczak, "Improving the accuracy of PGSE DTI experiments using the spatial distribution of $b$ matrix", Magnetic Resonance Imaging, vol. 33, no. 3, pp. 286-295, 2015, doi: 10.1016/j.mri.2014.10.007

[3] D. Güllmar, J. Haueisen, and J. R. Reichenbach, "Analysis of b-value calculations in diffusion weighted and diffusion tensor imaging," Concepts Magn. Reson., vol. 25A, no. 1, pp. 53-66, Mar. 2005, doi: 10.1002/cmr.a.20031

[4] J. Mattiello, P. Basser and D. Lebihan, "Analytical Expressions for the b Matrix in NMR Diffusion Imaging and Spectroscopy", Journal of Magnetic Resonance, Series A, vol. 108, no. 2, pp. 131-141, 1994, doi:10.1006/jmra.1994.1103

[5] J. Mattiello, P. Basser and D. Le Bihan, "The b matrix in diffusion tensor echo-planar imaging", Magnetic Resonance in Medicine, vol. 37, no. 2, pp. 292-300, 1997, doi: 10.1002/mrm. 1910370226

[6] Hsu EW, Mori S. Analytical expressions for the NMR apparent diffusion coefficients in an anisotropic system and a simplified method for determining fiber orientation. Magn Reson Med 1995;34:194-200, doi: $10.1002 / \mathrm{mrm} .1910370226$

[7] Neeman M, Freyer JP, Sillerud LO. A simple method for obtaining cross-term-free images for diffusion anisotropy studies in NMR microimaging. Magn Reson Med 1991;21:138-43, doi: $10.1002 / \mathrm{mrm} .1910210117$

[8] Güllmar D, Haueisen J, Reichenbach JR. Analysis of b-value calculations in diffusion weighted and diffusion tensor imaging. Concepts Magn Reson Part A 2005;25A:53-66. doi: 10.1002/cmr.a.20031

[9] S. Boujraf, R. Luypaert, H. Eisendrath and M. Osteaux, "Effect of accurately calculated $\mathrm{b}$ matrix on the evaluation of the diffusion tensor using echoplanar diffusion tensor imaging", ITBM-RBM, vol. 23, no. 6, pp. 340-344, 2002, doi:10.1016/S1297-9562(02)90003-3

[10] Ozcan A. Minimization of Imaging Gradient Effects in Diffusion Tensor Imaging. IEEE Trans Med Imaging 2011;30:642-54. doi:10.1109/TMI.2010.2090539

[11] [1] G. Nair and X. P. Hu, "Manifestation and Post-hoc Correction of Gradient Cross-term Artifacts in DTI," Magn Reson Imaging, vol. 30, no. 6, pp. 764-773, Jul. 2012, doi: 10.1016/j.mri.2012.02.021

[12] K. Borkowski and A. Krzyżak, "Simulations of rotation of the anisotropic phantom in BSD-DTI" in Magnetic Resonance Materials in Physics, Biology and Medicine, 2015 vol. 28 suppl. 1, s. 467-468. ESMRMB 2015 : 32nd annual scientific meeting : Edinburgh, UK, doi: $10.1007 / \mathrm{s} 10334-015-0490-7$

[13] P. Basher, J. Mattiello and D. Lebihan, "Estimation of the Effective Self-Diffusion Tensor from the NMR Spin Echo", Journal of Magnetic Resonance, Series B, vol. 103, no. 3, pp. 247-254, 1994, doi:10.1006/jmrb.1994.1037

[14] P. Kingsley, "Introduction to diffusion tensor imaging mathematics: Part II. Anisotropy, diffusion-weighting factors, and gradient encoding schemes", Concepts in Magnetic Resonance Part A, vol. 28, no. 2, pp. 123-154, 2006, DOI: 10.1002/cmr.a.20049

[15] A. Krzyżak and K. Borkowski, "Theoretical analysis of phantom rotations in BSD-DTI" in Engineering in Medicine and Biology Society (EMBC), 2015 37th Annual International Conference of the IEEE , vol., no., pp.410-413, 25-29 Aug. 2015, doi: 10.1109/EMBC.2015.7318386

[16] K. Kłodowski and A. Krzyżak, "Innovative anisotropic phantoms for calibration of diffusion tensor imaging sequences", Magnetic Resonance Imaging, vol. 34, no. 4, pp. 404-409, 2016, doi: 10.1016/j.mri.2015.12.010 